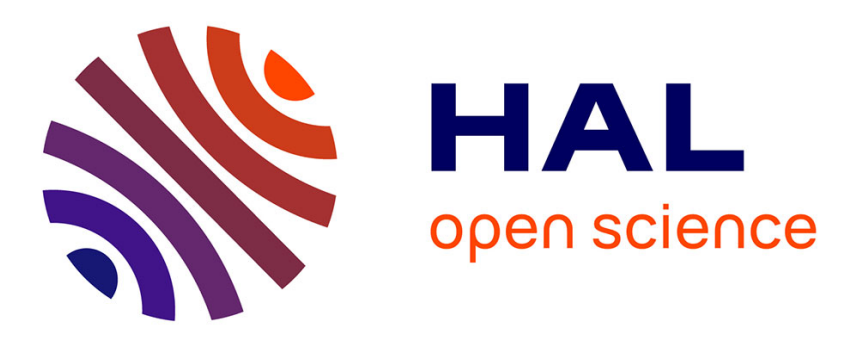

\title{
The use of reflection anisotropy spectroscopy to assess the alignment of collagen
}

a L Schofield, C I Smith, V R Kearns, D S Martin, T Farrell, P Weightman, R L Williams

\section{- To cite this version:}

a L Schofield, C I Smith, V R Kearns, D S Martin, T Farrell, et al.. The use of reflection anisotropy spectroscopy to assess the alignment of collagen. Journal of Physics D: Applied Physics, 2011, 44 (33), pp.335302. 10.1088/0022-3727/44/33/335302 . hal-00644268

\section{HAL Id: hal-00644268 \\ https://hal.science/hal-00644268}

Submitted on 24 Nov 2011

HAL is a multi-disciplinary open access archive for the deposit and dissemination of scientific research documents, whether they are published or not. The documents may come from teaching and research institutions in France or abroad, or from public or private research centers.
L'archive ouverte pluridisciplinaire HAL, est destinée au dépôt et à la diffusion de documents scientifiques de niveau recherche, publiés ou non, émanant des établissements d'enseignement et de recherche français ou étrangers, des laboratoires publics ou privés. 


\title{
The use of Reflection Anisotropy Spectroscopy to Assess the
}

\section{Alignment of Collagen}

\author{
A L Schofield ${ }^{1}$, C I Smith ${ }^{1}$, V R Kearns ${ }^{2}$, D S Martin ${ }^{1}$, T Farrell ${ }^{1}$, P Weightman ${ }^{1}$ and R L Williams ${ }^{2} *$ \\ ${ }^{1}$ Department of Physics, University of Liverpool, Liverpool, L69 7ZE, UK \\ ${ }^{2}$ Department of Eye and Vision Science, Institute of Ageing and Chronic Disease, University of \\ Liverpool, Liverpool, L69 3GA, UK \\ * Corresponding author
}

\begin{abstract}
The alignment of collagen fibres in tissue has a major influence on their mechanical properties. This study investigated the ability of Reflection Anisotropy Spectroscopy (RAS) to determine the degree of alignment of collagen fibres deposited onto surfaces and secreted by mouse fibroblast cells in vitro. Aligned nanofibres of polytetrafluoroethylene (PTFE) were deposited on glass coverslips using a simple friction transfer method. These linear parallel nanofibres were used as topographical cues to orientate and align L929 fibroblasts and their deposited collagen. The strength of the RAS signal was demonstrated to correlate with the degree of collagen alignment. Immunochemical staining and atomic force microscopy were used to visualise the topography of the fibres and confirm that the RAS signal was as a result of collagen fibres. Collagen deposited onto glass coverslips from a solution that had been subjected to dialysis that caused 'nanofibrillar' collagen to form also resulted in a strong RAS signal whereas collagen absorbed from a simple solution of collagen in which collagen fibres are not formed resulted in no RAS signal. It was concluded that the RAS signal could be used to determine the degree of alignment of collagen and that this could have a potential application in the assessment of collagen orientation in tissue repair.
\end{abstract}

PACS: 87.14.em; 87.64.Dz; 78.68.+m

\section{Introduction}


Collagen provides strength and stability to many human tissues including connective tissue, bone and tendon [1]. It has a diverse history as a biomaterial and in recent times composites based on collagen have been used in artificial bone substitutes [2] as well as the use of collagen-like proteins in matrices with hydroxyapatite as a framework for effective in-situ drug release [3]. Collagen has a hierarchical structure [4] and is shown in figure 1. The primary amino-acid sequence of the polypeptide chains is predominantly composed of glycine, proline, hydroxyproline and alanine with a smaller number of phenylalanine and tyrosine residues. The secondary structure is constructed from triple helices of the polypeptide chains that are stabilized by interchain hydrogen bonding [5]. The triple helical collagen (tropocollagen) molecules pack together to produce collagen microfibrils which ultimately aggregate to form macro-structural collagen fibres. The major collagen component in tendons is type I collagen and the major cell type is the fibroblast. The fibroblasts are sandwiched between the collagen fibres and oriented along the fibres. Their role is to synthesize extracellular matrix (ECM) proteins resulting in an organised collagen matrix and remodelling during tendon healing. It has been shown that in regular soft tissue, irregular organisation of collagen fibres leads to poor healing and repair [4].

a

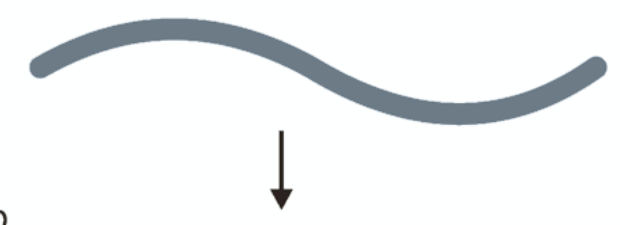

b

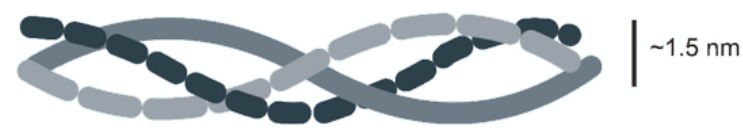

c $\quad \downarrow$
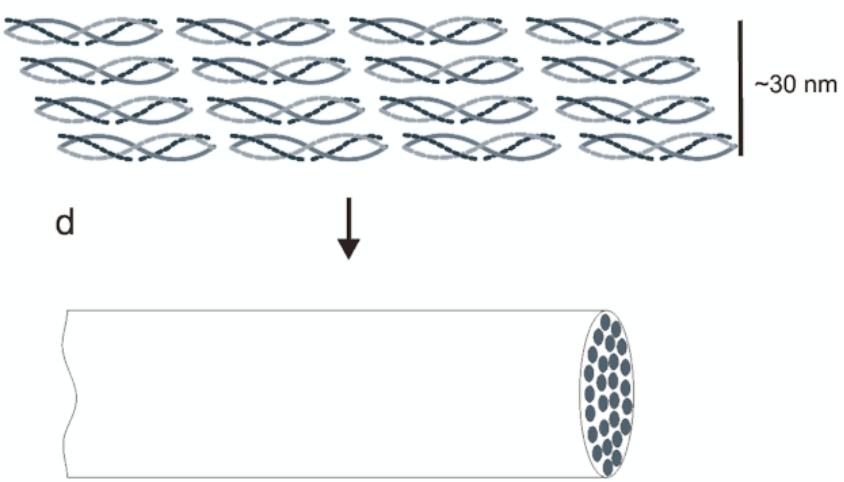
Figure 1. Diagrammatical representation of collagen structure (a) polypeptide molecule, (b) triple helical tropocollagen, (c) collagen fibrils and (d) collagen fibre.

In the development of effective biomaterials, the relationship between the cells and the surface of the material is important. Therefore, development of materials which can lead to correct organization and structure of tissue is crucial. It has been shown by many authors that chemical and topographic substrate surface patterning can be used as a method to control cellular organisation $[6,7]$. Over the past decade, nanomaterials have been shown to be good candidates for improving traditional tissue engineering materials [7]. The unique properties of nanomaterials have helped to improve the growth of tissue, as decreasing material size into the nanoscale increases surface area, roughness and surface area/volume ratio which can lead to superior physiochemical properties [8]. It has been shown that using nanofibre coated scaffolds potentially increases tissue regeneration in damaged human bladders due to the increased cell densities observed on the nanofibre coated scaffolds compared to uncoated scaffolds [9]. More recently human skeletal muscle cells seeded on to blended collagen nanofibre oriented meshes exhibited greater alignment and organization than those observed on randomly oriented nanofibre surfaces [10].

The influence of a nanopatterned surface on collagen orientation as well as the intrinsic anisotropy of collagen on both a microscopic and macroscopic scale makes it an ideal system for study with Reflection Anisotropy Spectroscopy (RAS). RAS could then be used as an analysis tool to determine the alignment of collagen in tissue repair and development of tissue engineering constructs. The RAS technique has been used to study metal surfaces [11-14], the orientation of molecules at metal/vacuum interfaces $[15,16]$, the orientation of biological molecules at metal/liquid interfaces [17-19] and polymer materials [20,21]. In the work presented here RAS was used to probe the influence of oriented nanofibres on glass substrates on the anisotropy and alignment of collagen produced by fibroblast cells in comparison to the anisotropy and alignment intrinsically present in collagen from solution and collagen which has been prepared using dialysis to form fibrils referred to herein as 'nanofibrillar' collagen. A large RAS signal was found related to the preferential alignment of collagen. 


\section{Experimental}

RAS is a linear optical technique that measures anisotropy at surfaces. In RAS, linearly polarised light illuminates the surface at normal incidence and the difference in reflectivity in two orthogonal directions of the surface is measured. For a cubic substrate this geometry results in cancellation of the bulk signal by symmetry and RAS becomes a probe of surface anisotropy. The technique was developed initially to probe the surfaces and growth of semiconductors [22,23] and has been recently reviewed [24].

The RA spectrometer used in this work follows Aspnes design [22] and the measured RA signal is given by

$$
\operatorname{Re}\left(\frac{\square r}{r}\right) \square \operatorname{Re}\left(\frac{2\left(r_{x} \square r_{y}\right)}{r_{x} \square r_{y}}\right)
$$

where $r_{\mathrm{x}}$ and $r_{\mathrm{y}}$ are the complex Fresnel reflection coefficient for the surface $x$ and $y$ direction respectively. The experiment was arranged such that the $x$ direction was parallel to the direction of the nanofibres and the $y$ direction perpendicular to the fibres. RA spectra of the real part of the complex RA were taken over a photon energy range of $1.5 \mathrm{eV}$ to $5.5 \mathrm{eV}$ with an illuminated sample area of $\sim 14 \mathrm{~mm}^{2}$. The angular variation of the RAS response was investigated by performing angular dependent RAS (ADRAS) [25]. The sample was rotated by an angle $\square$ about the plane of polarisation of the incident light and the RA spectrum recorded.

A simple friction transfer method [26] was used to deposit aligned PTFE (polytetrafluoroethylene) nanofibres onto borosilicate glass slides. A PTFE blade of $20 \mathrm{~mm}$ width and $1 \mathrm{~mm}$ thickness, bevelled at the edge to contact the glass slides, was used as the source of nanofibres. The glass slides were placed on a heated stage $\left(230^{\circ} \mathrm{C}\right)$ until they reached thermal equilibrium. The PTFE blade was brought into contact with the glass slide under a load of $1 \mathrm{~kg}$ and the slide was moved under the blade at a constant velocity to deposit linear nanofibres. Following the deposition, the glass slides were removed and allowed to cool in air. 
L929 murine fibroblasts cells were obtained from European Collection of Cell Cultures (ECACC). Cells were cultured at $37^{\circ} \mathrm{C}$ in a $5 \% \mathrm{CO}_{2}$ atmosphere in Dulbeccos Modified Eagles Medium (DMEM) (Sigma) supplemented with 5\% Foetal Bovine Serum (Invitrogen) and $1 \%$ Penicillin/Streptomycin antibiotics (Sigma) until they reached 60-70\% confluence. Culture medium was replenished at 2-3 day intervals.

The coverslips with and without the PTFE nanofibres were sterilised using ethanol and rinsed with ultra pure water and left to air dry overnight. The coverslips were irradiated with UV for $30 \mathrm{~min}$ to ensure sterility. Coverslips were then placed in each well of a tissue culture six well plate (Corning). L929 mouse fibroblast cell culture $\left(1 \times 10^{5}\right.$ cells/ml $)$ were seeded on each coverslip and incubated in a $5 \% \mathrm{CO}_{2}$ incubator at $37^{\circ} \mathrm{C}$ for $1,2,3$ and 4 week intervals. Culture medium was replenished at 2-3 day intervals. The plates were removed from incubation and refrigerated for approximately $30 \mathrm{~min}$. Coverslips were removed from the culture medium and washed with Dulbecco's Phosphate Buffered Saline (DPBS, Sigma). The cell layer was dispersed in $0.05 \%$ Trypsin/EDTA (Sigma) incubated for 3 min, and subjected to gentle agitation to remove the cells without damaging the collagen layer. Control samples were treated the same minus the trypsinisation step to maintain the cell layer. Coverslips were fixed with ice-cold methanol at $-18^{\circ} \mathrm{C}$ for $3-4$ minutes then stored in PBS at $4^{\circ} \mathrm{C}$ prior to further analysis.

Dry dialysis tubing was prepared to remove glycerol and sulfur compounds. Removal of glycerol was accomplished by washing the tubing in running water for 3-4 hours. Sulfur compounds were removed by treating with $0.3 \%(\mathrm{w} / \mathrm{v})$ solution of sodium sulfide at $80^{\circ} \mathrm{C}$ for 1 minute. The tubing was immersed into hot water $\left(60^{\circ} \mathrm{C}\right)$ for 2 minutes followed by acidification with a $0.2 \%(\mathrm{v} / \mathrm{v})$ solution of sulfuric acid this was then rinsed with fresh water to remove the acid. Preparation of the tubing in this way ensures that it retains most proteins of molecular weight $12000 \mathrm{kDa}$ (Spectra/Por ${ }^{\circledR}$ dialysis membranes molecular weight cut off 12,000 to $14,000 \mathrm{kDa}$ ) or greater which includes collagen type I, whilst allowing the passage of acid causing neutralisation of the solution. Fibrils have been shown to form from this dialysis method of the collagen solution against water at approximately $35^{\circ} \mathrm{C}[27]$. 
Purified collagen can be made to reconstitute in vitro into fibrils, referred to here as 'nanofibrillar collagen'. To create nanofibrillar collagen, solid collagen type I from calf skin (Sigma) was dissolved in $0.05 \%(\mathrm{v} / \mathrm{v})$ acidified water (acetic acid in water). Typically $5 \mathrm{mg}$ of collagen in $5 \mathrm{ml}$ of acidified water was made for each set of experiments. This was maintained between $2-8^{\circ} \mathrm{C}$ for 3 days and was then put into the dialysis tubing and placed in a large beaker of water which was left to stir overnight. The resultant collagen solution was then deposited onto borosilicate glass coverslips neat, and also at concentrations of 1:10, 1:100 and 1:1000 (v/v) in distilled water. The samples were left overnight to evaporate in air. The same dilutions of collagen from solution (Sigma) were also prepared and deposited on to the glass coverslips in the same way.

Methanol-fixed samples (unmodified glass coverslips or glass coverslips modified with PTFE nanofibres with either fibroblast derived collagen or fibroblast cells deposited upon the surface) were incubated with a rabbit anti-collagen I antibody (1:200 dilution; Abcam) or normal rabbit IgG (Caltag Laboratories) overnight at $4^{\circ} \mathrm{C}$, then for $1 \mathrm{~h}$ at $37^{\circ} \mathrm{C}$ with an Alexa Fluor ${ }^{\circledR} 594$ anti-rabbit secondary antibody (1:500 dilution; Invitrogen). Samples were mounted in Vectashield containing DAPI (Vector Laboratories LTD) and visualised using an LSM 500 laser scanning confocal microscope (Carl Zeiss).

AFM was performed using a Nanoscope III (Digital Instruments) in contact mode using silicon nitride probes of nominal spring constant $0.6 \mathrm{~N} / \mathrm{m}$. Images were obtained from the fibroblast secreted collagen on nanofibres, collagen deposited from solution and from the dialysis method, in all cases using borosilicate glass coverslips substrates. All images were obtained in ambient air after rinsing the samples with PBS and allowing to air dry.

\section{Results}

For glass coverslips, with and without the presence of aligned PTFE nanofibres, a relatively flat RAS profile was observed (figure 2). This result implies that the aligned PTFE nanofibres do not contribute any significant optical anisotropy to the RAS measurement. Thus RAS applied to these substrates will be surface sensitive and any anisotropy observed from collagen (or cells) deposited on these will result from the collagen (or cells). Similar RA spectra were obtained from fibroblast cells on glass

slides and fibroblasts on PTFE-nanofibre coated glass (figure 2). Both spectra exhibit a positive 
feature over the range $4.0 \mathrm{eV}$ to $5.5 \mathrm{eV}$. This signal may originate from the fibroblast cells or from collagen associated with the cells. The similarity of the signal in the two cases suggested that a similar degree of alignment was achieved for both these substrates. The RAS signal obtained from collagen deposited randomly onto glass was small and relatively flat across the spectral range.

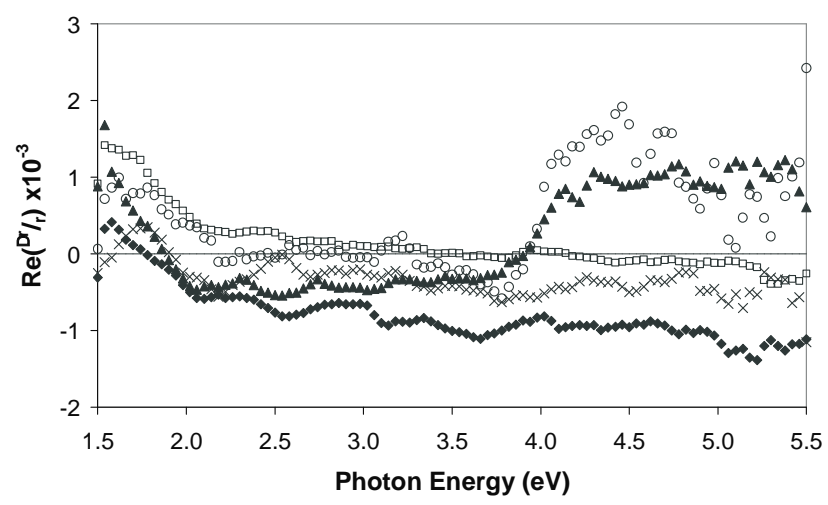

Figure 2. The RA spectra obtained at $\theta=45^{\circ}$ of borosilicate glass cover slips $+/$ - PTFE nanofibres. RA spectra are also shown for fibroblast cells grown for 1 week on glass and glass +/- PTFE nanofibres as well as fibroblast secreted collagen after 1 week on glass cover slips, obtained at $\square=45^{\circ}$ to the edge of the glass coverslip. Plain glass coverslips $(\times)$; PTFE $(\square)$; collagen on glass $(\bullet)$; fibroblasts on glass ( $\mathbf{A})$; fibroblasts on PTFE (O).

RAS signals obtained from fibroblast secreted collagen on PTFE coated glass coverslips (figure 3) were considerably larger than the RAS signals of the control surfaces. RA spectra obtained from fibroblast secreted collagen are shown at different sample rotation angles about the incident plane of light polarisation. The RAS response was relatively flat below $3.0 \mathrm{eV}$ whereas above $3.0 \mathrm{eV}$ there was a large signal which reaches $\sim 30$ units when the nanofibres are aligned along the RAS $x$ direction. The RAS data of unaligned collagen on glass (figure 2) showed a similar increase in RAS above $3.5 \mathrm{eV}$ although of a much smaller intensity. 


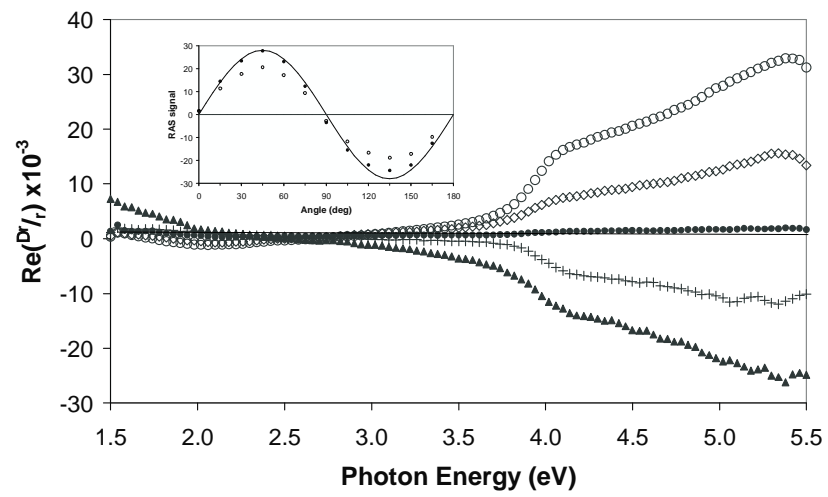

Figure 3. RA spectra obtained at $\theta=0^{\circ}(\bullet), 15^{\circ}(\diamond), 45^{\circ}(\bigcirc), 105^{\circ}(\times), 135^{\circ}(\triangle)$ showing minima, maxima and intermediate profiles for fibroblast secreted collagen after 1 week on PTFE coverslips. The insert shows ADRAS results for the same data set measured at $4.5 \mathrm{eV}(\bigcirc)$ and $5.0 \mathrm{eV}(\bullet)$. The solid line is $\cos (2 \square+\mathrm{f})$. The systematic error in the position of zero on the vertical scale of the order of $\pm 2 \times 10^{-3}$ RAS units.

The RA spectra obtained from solid collagen subjected to dialysis to create 'nanofibrillar' collagen diluted with water in a ratio of 1:100 (figure 4) showed a similar signal to that of the spectra obtained from fibroblast secreted collagen on nanofibres (figure 3), although of lesser intensity. RA spectra obtained from 'nanofibrillar' collagen are shown at different sample rotation angles about the incident plane of light polarisation. The RAS response is relatively small and flat below $3.0 \mathrm{eV}$ whereas above $3.0 \mathrm{eV}$ there is an increased signal which reaches $\sim 12$ units when the fibres are aligned along the RAS $x$ direction compared to $\sim 30$ units from fibroblast derived collagen on a nanofibre modified surface (figure 3). The ADRAS data are displayed in the insert of figures 3 and 4 . The ADRAS results for fibroblast-secreted collagen on nanofibres (figure 3) and dialysis-collagen (figure 4) show that in both cases the anisotropic RAS signal follows a simple $\cos [2(\square+\mathrm{f})]$ behaviour; where $\square$ is the angle between the nanofibre alignment direction and the linear polarisation direction of the incident light. The phase offset angle $\mathrm{f}=45^{\circ}$. For example, when $\square=45^{\circ}$ the long linear nanofibres are aligned along the $r_{\mathrm{x}}$ direction and the RAS signal reaches a maximum. ADRAS can be used to distinguish signals that arise from surface components that have different orientations of their 
optical axes. The simple rotation behaviour shown in figures 3 and 4 is consistent with the RAS signal arising from optical dipoles that have common optical axes

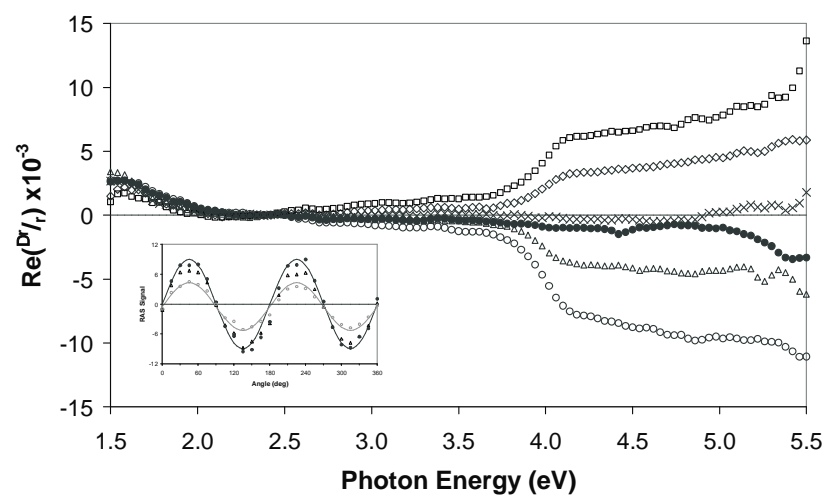

Figure 4. RA spectra obtained at $\theta=0^{\circ}(\bullet), 15^{\circ}(\diamond), 45^{\circ}(\square), 90^{\circ}(\times), 105^{\circ}(\triangle), 135^{\circ}(\bigcirc)$ showing minima, maxima and intermediate profiles for 'nanofibrillar' collagen derived from a dialysis method after deposition onto glass coverslips. The insert shows ADRAS results for the same data set measured at $4.0 \mathrm{eV}(\bigcirc)$ and $4.5 \mathrm{eV}(\triangle)$ and $5.0 \mathrm{eV}(\bullet)$. The systematic error in the position of zero on the vertical scale of the order of $\pm 2 \times 10^{-3}$ RAS units.

The RAS signal from 'nanofibrillar' collagen was compared to those obtained from collagen from solution (diluted to 1:100). RA spectra obtained from collagen solution deposited onto coverslips are shown at different sample rotation angles about the incident plane of light polarisation. This collagen on unmodified glass coverslips results in a relatively flat RAS profile (figure 5).

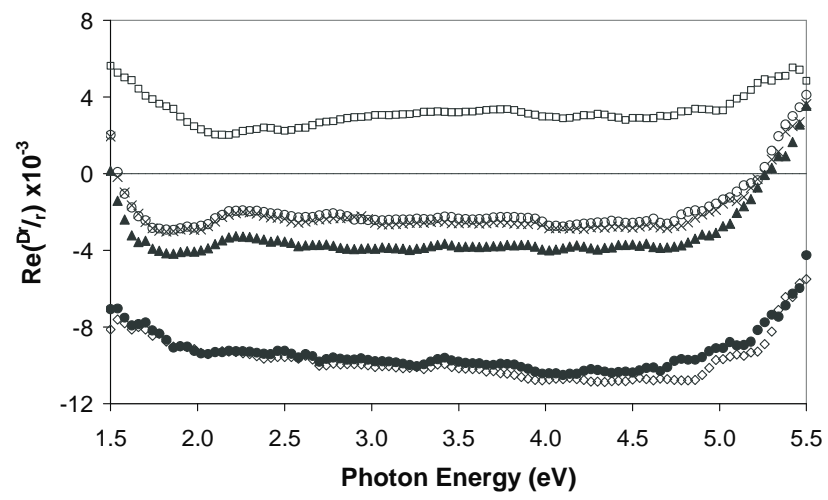

Figure 5. RA spectra obtained at $\square=0^{\circ}(\bullet), 15^{\circ}(\diamond), 45^{\circ}(\square), 90^{\circ}(\times), 105^{\circ}(\bigcirc), 135^{\circ}(\mathbf{\Delta})$ showing minima, maxima and intermediate profiles for a 1:100 dilution of collagen from solution after 
deposition onto glass coverslips. The variation in the level of the RAS signal as a function of angle is a real effect probably arising from the $\sin \square$ dependence in ADRAS measured noted by Lane et al [28].

Immunochemical visualisation of collagen was performed on samples where collagen had been secreted by fibroblasts and aligned using nanofibres as well as those samples where collagen had been secreted by cells on glass without nanofibres. Investigation using confocal microscopy revealed the presence of collagen confined in particular regions which confirms preferential alignment of collagen in the direction of the nanofibres (figure 6(b)), compared to those samples without nanofibres (figure 6(a)). The negative control using rabbit $\operatorname{IgG}$ on surfaces with cells present or removed and on nanofibre and plain glass surfaces did not show immuno-reactivity with any collagen present (data not shown).
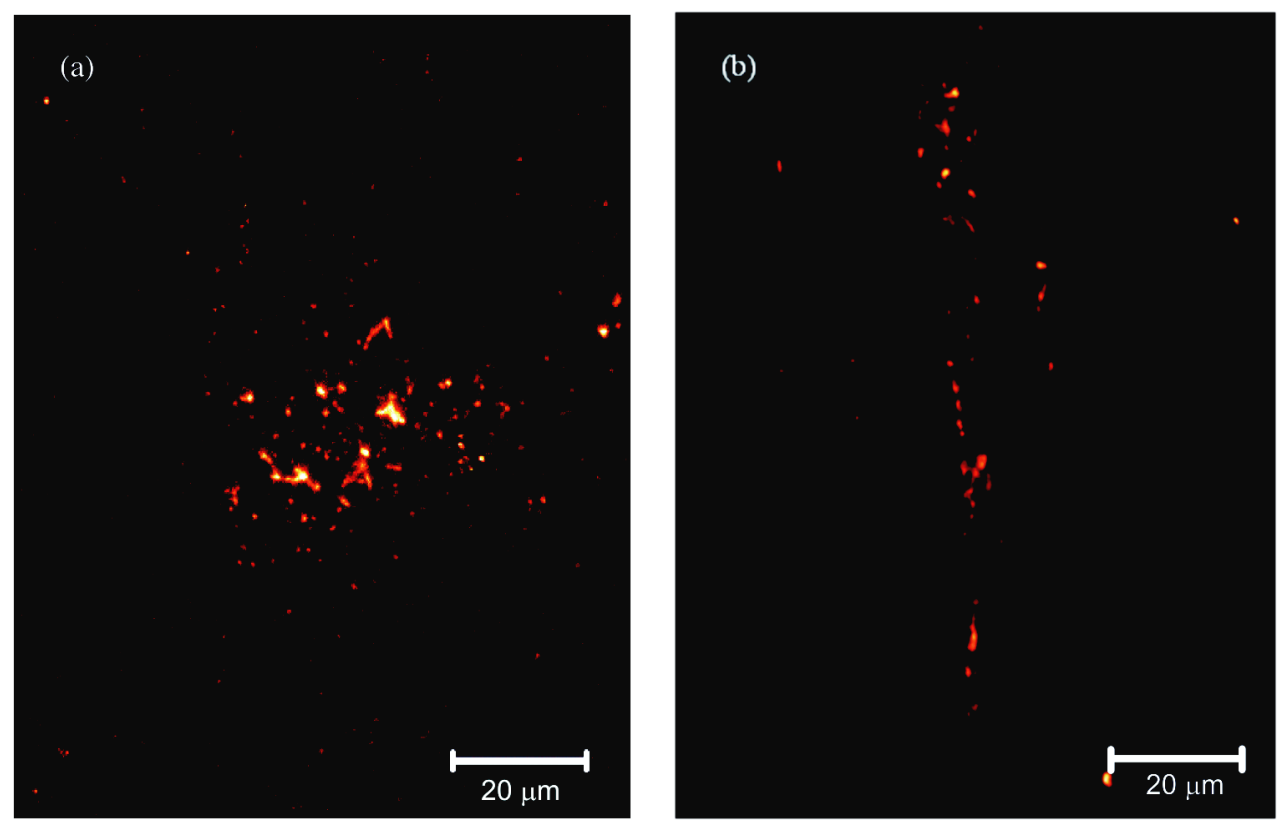

Figure 6. Immunochemical visualisation of fibroblast derived collagen on (a) unpatterned borosilicate glass slide demonstrating random orientation and (b) PTFE nanofibres demonstrating directional orientation. Image colour has been enhanced with 'glow scale' feature of LSM image browser (Carl Zeiss). Scale bar $=20 \mu \mathrm{m}$ on both images.

Atomic force microscope investigation of fibroblast secreted collagen on PTFE nanofibres also revealed alignment of material along the direction of the nanofibres. Figure 7 shows an area on a 
PTFE nanofibres-coated coverslip where features (raised whiter areas) can be seen aligned along the long axis of a PTFE nanofibre(s). Material assumed to be collagen can be seen on the glass surfaces in between the nanofibres and on the nanofibres.

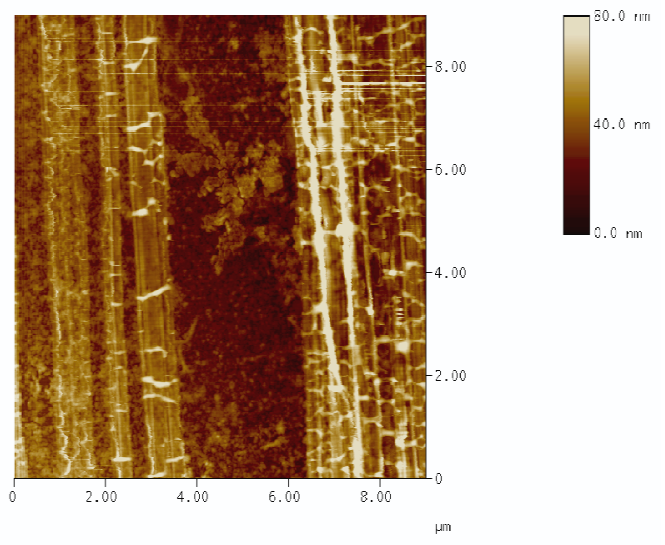

Figure 7. $9 \mu \mathrm{m}$ by $9 \mu \mathrm{m}$ AFM image of fibroblast secreted collagen on nanofibres.

AFM revealed different images of 'nanofibrillar' collagen and collagen deposited onto glass coverslips from solution. The AFM image obtained from collagen 'solution' (figure 8(a)) demonstrates the non-uniform deposition of collagen from solution onto the surface. The features in the image are typically $\sim 30 \mathrm{~nm}$ in height, significantly larger than the typical height of $5 \mathrm{~nm}$ of the nanofibrillar collagen (in figure 8(a) and (b) respectively)). The 'nanofibrillar' collagen deposited onto glass (figure $8(\mathrm{~b})$ ) revealed coverage of strand like features indicating the presence of collagen fibrils.
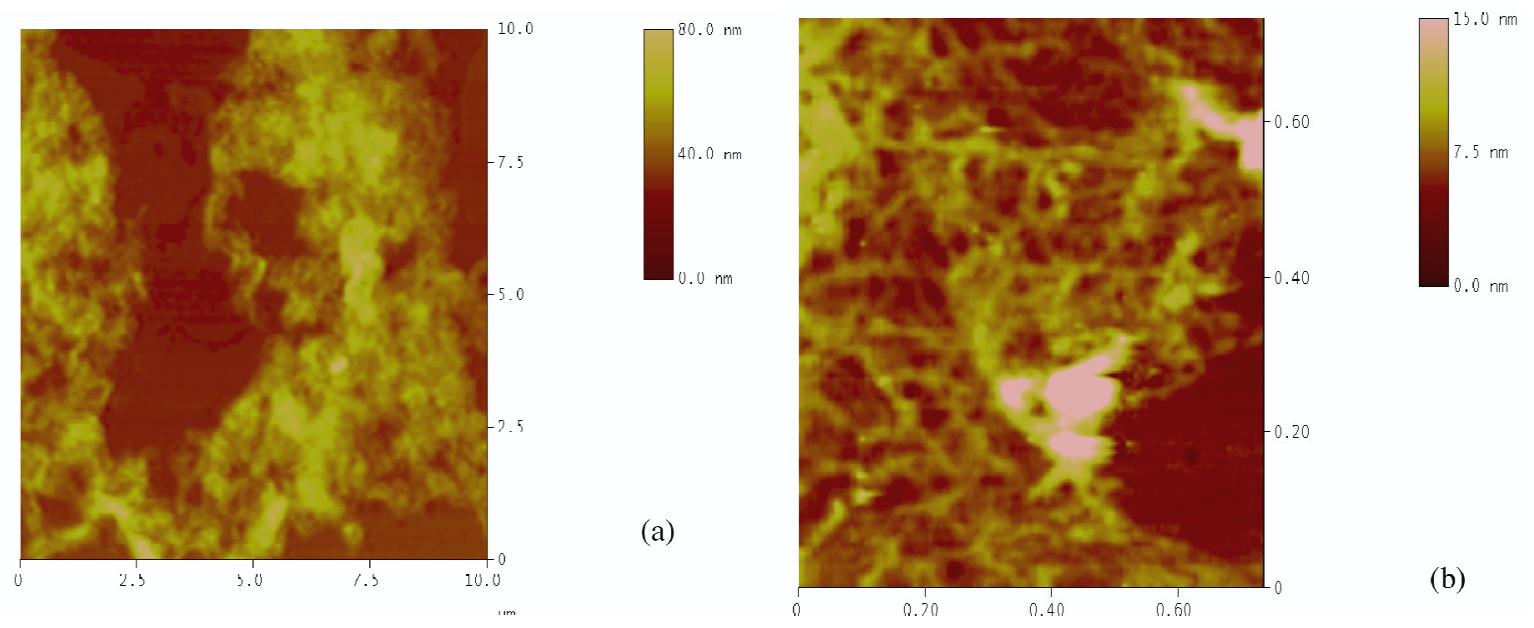

(b) 
Figure 8. (a): $10 \mu \mathrm{m} \times 10 \mu \mathrm{m}$ image of 1:100 collagen from solution deposited on plain glass coverslips and (b): $0.8 \mu \mathrm{m} \times 0.8 \mu \mathrm{m}$ image of 1:100 'nanofibrillar' collagen from the dialysis method deposited on plain glass coverslips.

\section{Discussion}

RAS is a very sensitive microscopic probe of macroscopic anisotropy. The optical signal arises from optical dipoles on the molecule length scale. However if these dipoles are orientated perfectly randomly on a surface then the RAS signal will be zero due to the cancellation of the net optical response in each direction. Thus macroscopic anisotropy is required in order to yield a non zero RAS signal and the strength of the signal is proportional to the degree of macroscopic anisotropy.

In order to assess the value of RAS as a tool in monitoring the alignment of collagen it is first necessary to establish the RAS profile of collagen. The flat RAS response of the glass coverslips and aligned PTFE nanofibres establish that the RAS signals observed in this work arise from either collagen or from fibroblast cells (figure 2). The RAS profiles obtained from collagen secreted by fibroblasts on PTFE coated glass coverslips (figure 3) and 'nanofibrillar' collagen have identical shapes that are essentially zero up to $\sim 3.8 \mathrm{eV}$ and then increase to higher energy. The RAS profiles also have identical azimuthal angular variations but very different intensities (figure 3 and 4). Since the method of preparation of the nanofibrillar collagen specimens exclude the possibility of a contribution from fibroblast cells it is clear that the RAS profiles of figures 3 and 4 arise from collagen. This conclusion is supported by visualisation of the presence of collagen using immunochemical staining and the fibre topography by atomic force microscopy.

The absence of a RAS signal from specimens formed by adsorption from a solution of dissolved collagen in which collagen fibres are not formed is expected since this method of preparation is unlikely to produce a net anisotropy in the alignment of collagen on the surface. The strong RAS signal observed from 'nanofibrillar' collagen is consistent with the AFM results which show that, in contrast to the specimens formed from the solution of dissolved collagen, these specimens consistent of linear strand like features. Although the AFM results do not indicate a preferred direction of the nanofibrils it is unlikely that the sum of the alignments cancels exactly 
giving rise to a net anisotropy. The AFM images of the collagen deposited on the linear parallel PTFE nanofibres shows a significant anisotropy which is consistent with the strong RAS signal. The alignment of collagen along the direction of the nanofibres is shown by (figure 7) the topographically higher features in the image. In addition, perpendicular structures were also observed across the direction of the fibres (figure 7). These perpendicular structures may be due to how collagen associates to form fibrils. The perpendicular fibre arrangement may reduce the overall RAS intensity as they will subtract from the RAS signal. Collagen fibrils associate in many tissues to form discrete fibre structures. Within one collagen fibre, the fibrils are oriented not only longitudinally but also transversely and horizontally. The longitudinal fibres do not run only parallel but also cross each other forming spirals $[29,30]$. A possible explanation for this perpendicular feature could be the presence of what are known as fibrous long spacing collagen fibrils (FLS). These are collagen fibrils that display a typical periodicities in the range from 150 to $250 \mathrm{~nm}$ [29,31]. Work carried out by Rasmusson et al. in 1994 [32] demonstrates the adsorption of protein macromolecules (fibrinogen) on to PTFE nanofibres and argues that adsorption of proteins on to hydrophobic surfaces causes the molecule to change its conformation to expose hydrophobic regions to the hydrophobic surface. In this work perpendicular molecule orientation to fibre direction was observed. Rasmusson et al. [32] reports that if the curvature radius of the fibre is small enough it will be more favourable for the molecule to adsorb perpendicular to the fibre direction as this will not require extra conformational change to obtain the same contact area. This could be a possible explanation for the perpendicular features observed when fibroblast derived collagen adsorbed on PTFE nanofibres as observed in the AFM images (figure 7). It is clear from the above that collagen has a characteristic RAS profile the intensity of which has been demonstrated to correlate with the degree of collagen alignment.

RAS is known to be very sensitive to surface topography [24]. It is known that surface topography influences cell attachment and orientation and RAS could be useful to probe these interactions. Cell-substratum interactions are key to many biological processes and this is important to the design of medical devices and in tissue engineering. Responses of cells to topographical cues and the concept of contact guidance are well known. A wide variety of topographical features have been 
presented to cells including fibroblasts and topography has been shown to influence cellular response from initial attachment to differentiation and production of new tissue [33]. It has long been known that cells respond to surface topography, this is the same for nanotopography as it would be for other scales [7]. Specifically fibroblast cells have been shown to respond to many different types of surface patterning in the past $[34,35]$. Fibroblasts cells probe the substrate using nanometre scale filopedia presented on the cell lamellae $[7,36]$. It is therefore likely that they will be influenced by nanoscale patterning. Collagen type I is the primary constituent of the Extra Cellular Matrix (ECM) of fibroblast cells and it has been shown elsewhere that nanometer level surface features can be used to control matrix organisation. Manwaring et al. [37] engineered meningeal cell asymmetry in order to manipulate the ECM of these cells in culture. It was found that cell morphology was affected by nanometre topography and alignment increased with increasing surface roughness and in addition linear arrays of ECM were expressed that appeared to be related to cell orientation. This work demonstrates that the scarring response, which is inhibitory to the organised healing of the ECM in injured tendons can be interfered with by surface nanopatterns with at least one dimension smaller than the size of the material of interest either protein molecules or cells stimulating different molecular or cell behaviour. Work done by Denis et al. [38] reports the use of anisotropic, flat surface chemical nanopatterns to direct collagen adsorption. In this AFM study, spontaneous collagen adsorption on these chemically patterned substrates resulted in the accumulation of collagen on the hydrophobic tracks which is influenced by the width of the tracks which was smaller than that of the collagen molecules. Similarly work done by Lee et al. [39] showed that mechanical loading can alter fibroblast populated collagen fibre tissue constructs on already anisotropic surfaces. The highly organised nature of the collagen in tendons is responsible for the biomechanical properties of tendons. Whilst injured tendons heal naturally, scarring makes them weaker than uninjured tendon. The loss of mechanical competency is attributed to irregular orientation of collagen in the ECM [4]. Assessing organisation of collagen fibres in the ECM of tissues is therefore crucial in developing tissue engineering strategies and the development of biomaterials. RAS could offer the ability to assess the efficacy of differing tissue engineering strategies such as modification of surface topography but also assessing alignment as a result of mechanical stimulation. 


\section{Conclusion}

This study has demonstrated that the optical RAS technique can be used to characterise the degree of alignment of collagen when adsorbed on a surface. Dissolved collagen that had no fibrous component did not produce a RA spectrum. 'Nanofibrillar' collagen produced by a dialysis method produced a weak RA spectrum for collagen which correlated with the random arrangement of the collagen fibrils. Collagen secreted by fibroblasts aligned by an oriented surface topography produced a strong RA spectrum with the same features. It is concluded that there is a potential to develop RAS to study the alignment of collagen which could be useful in the evaluation of tissue repair and tissue growth in tissue engineering constructs.

\section{Acknowledgements}

We acknowledge the Engineering and Physical Sciences Research Council (EP/E046088/1), UK, for sponsoring this work.

\section{References}

[1] Falini G, Fermani S, Foresti E, Parma B, Rubini K, Sidoti M C and Roveri N 2004 J. Mater. Chem. 142297

[2] Sato K, Kumara Y and Tanaka J 1999 J. Biomed. Mater. Res. 5016

[3] Tampieri A, Celotti G, Landi E and Montevecchi M 2003 J. Mater. Sci.-Mater. Med. 14623

[4] Wang J H C 2006 J. Biomech. 391563

[5] Sionkowska A 2006 J. Photochem. Photobio. B-Biol. 8438

[6] Dalby M J, Giannaras D, Riehle M O, Gadegaard N, Affrosman S and Curtis A S G 2004 Biomaterials 2577

[7] Ansleme K, Ploux L and Ponche A 2010 J. Adhes. Sci. Technol. 24831

[8] Zhang L and Webster T J 2009 Nano Today 466

[9] Harrington D A, Cheng E Y, Guler M O, Lee L K, Donovan J L, Claussen R C and Stupp S I 2006

J. Biomed. Mater. Res. Part A 78A 157 
[10] Choi J S, Lee S J, Christ G J, Atala A and Yoo J J 2008 Biomaterials 292899

[11] Isted G E, Martin D S, Marnell L and Weightman P 2004 Surf. Sci. 566-568 35

[12] Sun L D, Hohage M, Zeppenfed P and Balderas-Navarro R E 2005 Surf. Sci. 589, 153

[13] Stanhrenberg K, Herrmann Th., Esser N and Richter W 2001 Phys. Rev. B 65, 034507

[14] Smith C I, Bowfield A, Almond N J. Mansley C P, Convery J H and Weightman P 2010 J. Phys.: Condens. Matter 22, 392001

[15] Frederick B G, Cole R J, Power J R, Perry C C, Chen Q, Richardson N V, Weightman P, Verdozzi C, Jennison D R, Schultz P A and Sears M P 1998 Phys. Rev. B 5810883

[16] Bussetti G, Cirilli S, Violante A, Chiaradia, Goletti C, Tortora L, Paolesse R, Martinelli E, D’Amico A, Di Natale C, Giancane G and Valli L 2009 Appl. Phys. Lett. 95, 091906

[17] Weightman P, Dolan G J, Smith C I, Cuquerella M C, Almond N J, Farrell T, Fernig D G, Edwards C and Martin D S 2006 Phys. Rev. Lett. 96086102

[18] Cuquerella M C, Smith C I, Fernig D G, Edwards C and Weightman P 2007 Langmuir 232078

[19] Smith C I, Bowfield A, Dolan G J, Cuquerella M C, Mansley C P, Fernig D G, Edwards C and Weightman P 2009 J. Chem. Phys. 130044702

[20] Farrell T, Smith C I, Schofield A L, Williams R L and Weightman P 2010 J. Phys. D: Appl. Phys. 43245301

[21] MacDonald B F and Cole R J 2003 J. Phys. D: Appl. Phys. 36142

[22] Aspnes D E, Harbison J P, Studna A A and Florez L T 1988 J. Vac. Sci. Technol. A 61327

[23] Kamiya I, Aspnes D E, Tanaka H, Florez L T, Harbison J P and Bhat R 1992 Phys. Rev. Lett. 68 627

[24] Weightman P, Martin D S, Cole R J and Farrell T 2005 Rep. Prog. Phys. 681251

[25] McDonald B F, Law J S and Cole R J 2002 J. Appl. Phys. 936

[26] Kearns V, Doherty P, Beamson G, Martin N and Williams R L 2010 J. Mater. Sci.-Mater. Med. 212213

[27] Lyons T E 2009 Liverpool University Ph.D. Thesis

[28] Lane P D, Isted G E, Roseburgh D S and Cole R J 2009 Appl. Phys. Lett. 95, 141907

[29] Wess T J 2005 Adv. Protein Chem. 70341 
[30] Kannus P 2000 Scand. J. Med. Sci. Spor. 10321

[31] Paige M F, Rainey J K and Goh M C 2001 Micron 32341

[32] Rasmusson J R, Erlandsson R, Salanek W R, Schott M, Clark D T and Lundstom I 1994 Scanning Microsc. 8481

[33] Yim E K F, Reano R M, Pang S W, Yee A F, Chen C S and Leong K W 2005 Biomaterials 26 5405

[34] Dalby M J, Childs S, Riehle M O, Johnstone H J H, Affrossman S and Curtis A S G 2003 Biomaterials 24927

[35] Dalby M J, Riehle M O, Yarwood S J, Wilkinson C D W and Curtis A S G 2003 Exp. Cell. Res. 284274

[36] Dalby M J, Riehle M O, Sutherland D S, Agheli H and Curtis A S G 2004 Biomaterials 255415

[37] Manwaring M E, Walsh J F and Tresco P A 2004 Biomaterials 253631

[38] Denis F A, Pallandre A, Nysten B, Jonas A M and Dupont-Gillain C C 2005 Small 1984

[39] Lee E J, Holmes J W and Costa K D 2008 Ann. Biomed. Eng. 361322 